\title{
Observation of Proton Transfer Coupled Spin Transition and Trapping of Photoinduced Metastable Proton Transfer State in an Fe(II) Complex
}

Takumi Nakanishi ${ }^{1}$, Yuta Hori ${ }^{2}$, Hiroyasu Sato ${ }^{3}$, Shuqi $\mathrm{Wu}^{1}$, Atsushi Okazawa ${ }^{4}$, Norimichi Kojima ${ }^{5}$, Takashi Yamamoto $^{6}$, Yasuaki Einaga ${ }^{6}$, Shinya Hayami ${ }^{7,8}$, Yusuke Horie ${ }^{9}$, Hajime Okajima ${ }^{9}$, Akira Sakamoto ${ }^{9}$, Yoshihito Shiota $^{1}$, Kazunari Yoshizawa ${ }^{1}$, and Osamu Sato ${ }^{1 *}$

${ }^{1}$ Institute for Materials Chemistry and Engineering \& IRCCS, Kyushu University, 744 Motooka, Nishi-ku, Fukuoka 819-0395, Japan.

${ }^{2}$ Center for Computational Sciences, University of Tsukuba, Tsukuba 305-8577, Japan.

${ }^{3}$ Rigaku Corporation, 3-9-12 Matsubaracho, Akishima, Tokyo 196-8666, Japan.

${ }^{4}$ Department of Basic Science, Graduation School of Arts and Sciences, The University of Tokyo, 3-8-1 Komaba, Meguro-ku, Tokyo 153-8902, Japan.

${ }^{5}$ Toyota Physical and Chemical Research Institute, Yokomichi, Nagakute, Aichi 480-1192, Japan.

${ }^{6}$ Department of Chemistry, Faculty of Science and Technology, Keio University, 3-14-1 Hiyoshi, Kohoku-ku, Yokohama, Kanagawa 223-8522, Japan.

${ }^{7}$ Department of Chemistry, Faculty of Advanced Science and Technology, Kumamoto University, 2-39-1 Kurokami, Chuo-ku, Kumamoto 860-8555, Japan.

${ }^{8}$ Institute of Pulsed Power Science (IPPS), Kumamoto University, 2-39-1 Kurokami, Chuo-ku, Kumamoto 860-8555, Japan.

${ }^{9}$ Graduate School of Science and Engineering, Aoyama Gakuin University, 5-10-1 Fuchinobe, Chuo-ku, Sagamihara, Kanagawa 252-5258, Japan.

*Corresponding author. Email: sato@cm.kyushu-u.ac.jp 


\section{Materials and Methods}

\section{Materials}

All reagents and solvents used in this study were purchased from TCI Co., Ltd., Wako Pure Chemical Industries Ltd., Alfa Aesar., and Sigma-Aldrich Chemical Co., and used without further purification.

Synthesis of $N^{\prime}$-(di(pyridin-2-yl)methylene)-2-chlorobenzohydrazide (HL-Cl).

Di-2-pyridyl ketone (1.84 g, $10 \mathrm{mmol})$ and 2-chlorobenzohydrazide $(1.70 \mathrm{~g}, 10 \mathrm{mmol})$ were added to ethanol $(100 \mathrm{ml})$. The mixture was refluxed for $12 \mathrm{~h}$ and left to stand at room temperature for slow evaporation of the solvent. White fluffy crystals of $\mathrm{HL}-\mathrm{Cl}$ were obtained after one week. Anal. $\mathrm{C}_{18} \mathrm{H}_{13} \mathrm{ClN}_{4} \mathrm{O}$ (336.78); calcd. C 64.20, H 3.89, N 16.64; found C 64.22, H 3.76, N 16.69.

Synthesis of $N^{\prime}$-(di(pyridin-2-yl)methylene)-2-methoxybenzohydrazide (HL-OMe).

HL-OMe was synthesized using the same procedure as described above for HL-Cl except that 2methoxybenzohydrazide was used instead of 2-chlorobenzohydrazide. Colorless plate-shaped crystals of HLOMe were obtained after three days. Anal. $\mathrm{C}_{19} \mathrm{H}_{16} \mathrm{~N}_{4} \mathrm{O}_{2}$ (332.3); calcd. C 68.66, $\mathrm{H}$ 4.85, N 16.86; found C 68.37, H 4.89, N 16.81.

Synthesis of $\left[\mathrm{Fe}(\mathrm{HL}-\mathrm{OCl})_{2}\right]\left(\mathrm{AsF}_{6}\right)_{2}([\mathbf{1 C l}])$.

Warning: Arsenates are toxic and should be handled with extreme care.

$\mathrm{HL}-\mathrm{Cl}(204 \mathrm{mg}, 0.6 \mathrm{mmol})$ and $\mathrm{FeCl}_{2} \cdot 4 \mathrm{H}_{2} \mathrm{O}(59 \mathrm{mg}, 0.3 \mathrm{mmol})$ were dissolved in methanol (15 ml) under a nitrogen atmosphere. $\mathrm{LiAsF}_{6}(114 \mathrm{mg}, 0.6 \mathrm{mmol})$ was then added to the methanol solution, and the resultant solution was capped and left to stand at room temperature for three days, affording black plate-shaped crystals of [1Cl] (144 mg, 43\%). $\mathrm{C}_{36} \mathrm{H}_{26} \mathrm{As}_{2} \mathrm{Cl}_{2} \mathrm{~F}_{12} \mathrm{FeN}_{8} \mathrm{O}_{2}$ (1107.23); calcd. C 39.05, H 2.37, N 10.12; found C 39.21, H 2.41, N 10.14.

Synthesis of $\left[\mathrm{Fe}(\mathrm{HL}-\mathrm{OMe})_{2}\right]\left(\mathrm{ClO}_{4}\right)_{2}([\mathbf{1 O M e}])$.

Warning: Perchlorate salts are potentially explosive and should be handled with extreme care.

HL-OMe (35 mg, $0.1 \mathrm{mmol})$ was dissolved in ethanol $(45 \mathrm{ml})$ under a nitrogen atmosphere. Then, $\mathrm{Fe}\left(\mathrm{ClO}_{4}\right)_{2} \cdot 6 \mathrm{H}_{2} \mathrm{O}(18 \mathrm{mg}, 0.05 \mathrm{mmol})$ was added to the ethanol solution, and the resultant solution was capped and allowed to stand in dry oven at $60^{\circ} \mathrm{C}$. Black plate-like crystal of [10Me] were obtained after three days (20 mg, 43\%). $\mathrm{C}_{38} \mathrm{H}_{32} \mathrm{Cl}_{2} \mathrm{FeN}_{8} \mathrm{O}_{12}$ (919.46); calcd. C 49.64, H 3.51, N 12.19; found C 49.49, H 3.42, N 12.19.

\section{X-ray structure determination}

All single crystals were coated with oil base cryoprotectant and mounted on nylon loops. Diffraction data were collected at and above $100 \mathrm{~K}$, and at $25 \mathrm{~K}$ under a cold nitrogen or helium gas stream, respectively, on a Rigaku CCD or an HPC X-ray diffractometer, using multi-layer mirror monochromated Mo-K $\alpha$ radiation $(\lambda$ $=0.71073 \AA$ ). Intensity data were collected at 180 and $350 \mathrm{~K}$ for [1CI]; and at 150 and $300 \mathrm{~K}$ for [10Me] using a $0.5^{\circ}$ oscillation angle. After the $25 \mathrm{~K}$ data collections for [1Cl], the crystals were irradiated with $532 \mathrm{~nm}$ light (GLMP-0200A) for $1 \mathrm{~h}$ and subjected to additional data collection. Bragg spots were integrated using the CrysAlis ${ }^{\text {Pro }}$ program package, and the empirical absorption correction (multi-scan) was applied using the SCALE3 ABSPACK program. Structures were solved by direct methods (SHELX-S ver. 2013/1 and SHELXT Version 2014/4) and refined by full-matrix least squares (SHELXL Version 2018/1). Hydrogen atoms that coordinated with carbon atoms in all crystal structures and those that coordinated with nitrogen atoms in the $[\mathbf{1 C l}]$ crystal structure at $25 \mathrm{~K}$ before and after irradiation were placed at calculated positions, and the riding models were refined and reapplied. The hydrogen atoms that coordinated with nitrogen atoms in the [1Cl] at 180 and $350 \mathrm{~K}$, and [1OMe] at 150 and $300 \mathrm{~K}$ were placed on the basis of Fourier map differences. 
CCDC1832822 ([1Cl] $350 \mathrm{~K}), 1832823$ ([1Cl] $180 \mathrm{~K}), 1832824([\mathbf{1 C l}] 25 \mathrm{~K}), 18328125([\mathbf{1 C l}] 25 \mathrm{~K}$ after irradiation), 1832826 ([1OMe] $300 \mathrm{~K})$ and 1832827 ([1OMe] $150 \mathrm{~K}$ ) contain the supplementary crystallographic data for this paper. These data can be obtained free of charge via http://www.ccdc.cam.ac.uk/conts/retrieving.html or from the CCDC (12 Union Road, Cambridge CB2 1EZ, UK; Fax: +44 1223 336033; E-mail: deposit@ccdc.cam.ac.uk).

\section{UV-vis spectroscopy}

We measured the temperature-dependent UV-vis absorption spectra of [1Cl] using a UV-3100PC (Shimadzu) scanning spectrophotometer with a helium-flow-type refrigerator. Samples were prepared by pasting the compound onto colorless tape. 


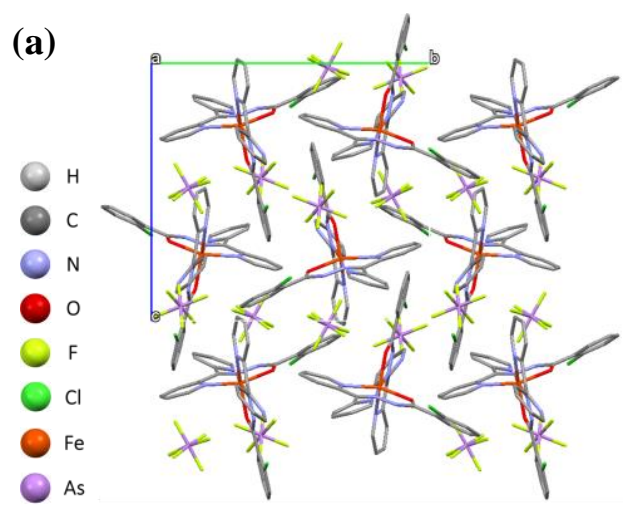

(b)

(c) (A)
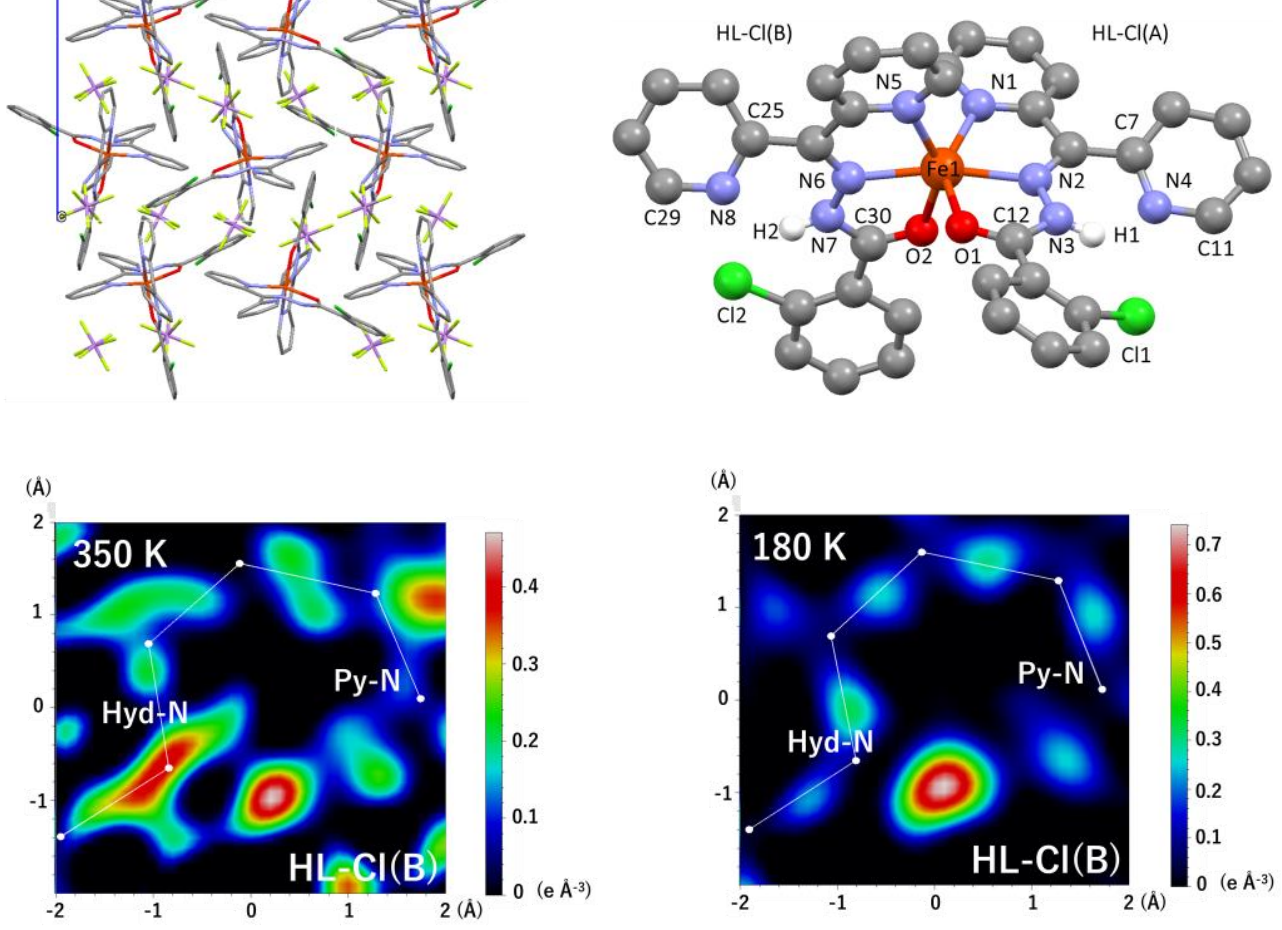

Figure S1. (a) Overview of the crystal structure of [1Cl] at $350 \mathrm{~K}$, as viewed parallel to the $a$-axis. (b) Structure of complex [1Cl] with labeled atoms involved either in the coordination with Fe(II) or in intramolecular hydrogen bonds. (c) Difference Fourier maps of $[1 \mathrm{Cl}]$ around the intramolecular hydrogen bond in $\mathrm{HL}-\mathrm{Cl}(\mathrm{B})$ at 350 and $180 \mathrm{~K}$. The standard deviations of the electron density in the difference Fourier map at 350 and $180 \mathrm{~K}$ were 0.1 and 0.07 , respectively. The maximum electron density at the expected

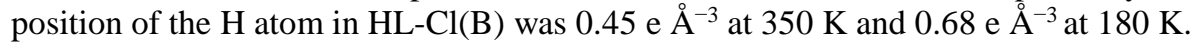

(a)

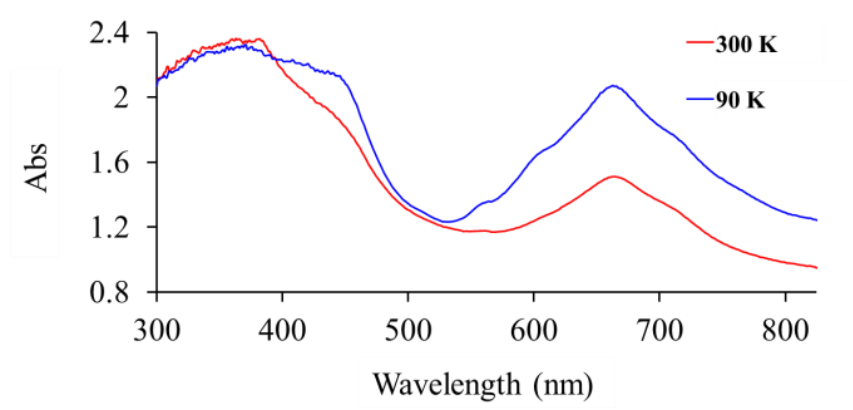

(b)

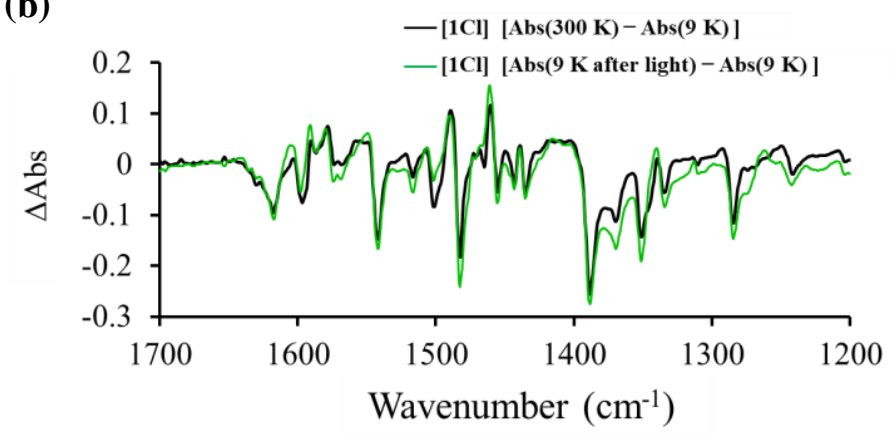

(c)

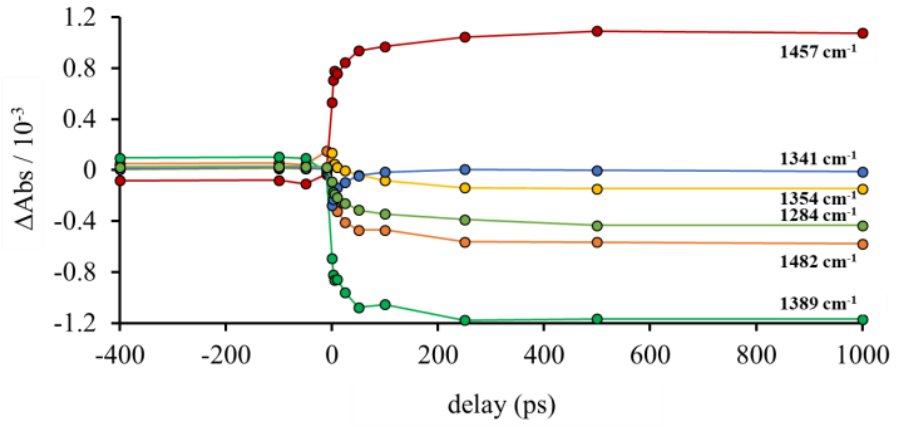

Figure S2. (a) Absorption spectra of [1Cl] at $300 \mathrm{~K}$ (red) and $90 \mathrm{~K}$ (blue). The broad peaks centered at around 360 and $660 \mathrm{~nm}$ are assigned as the ligand-based transition and metal-to-ligand charge-transfer transition, respectively. (b) IR difference spectrum for [1Cl] between the HS state at $300 \mathrm{~K}$ and the LS state at $9 \mathrm{~K}$ (black line) and that between the light-induced state at $9 \mathrm{~K}$ and the LS state at $9 \mathrm{~K}$ (green line). (c) Time-resolved IR absorption intensities of [1Cl] plotted against the delay time between the pump and probe pulses. 
(a)

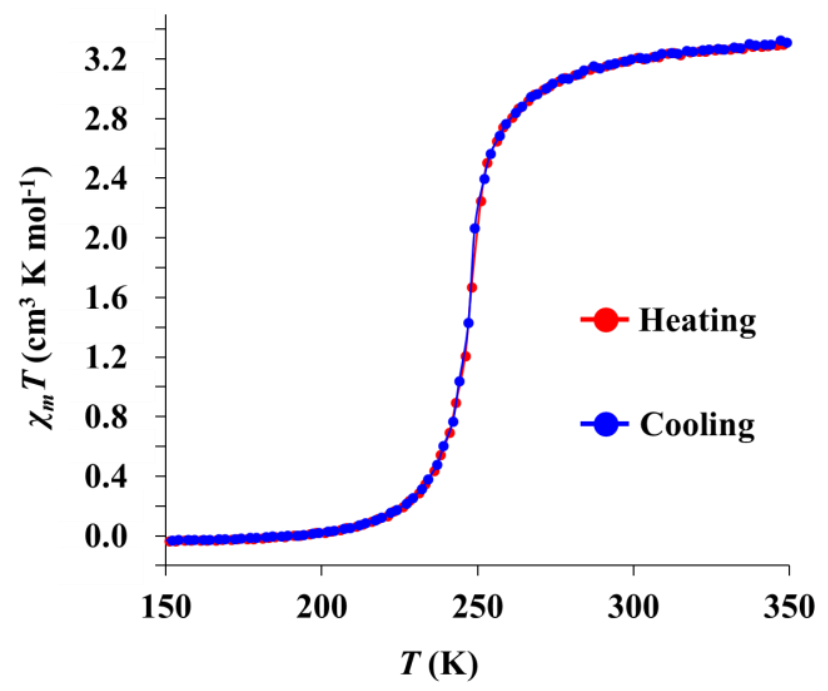

(b)

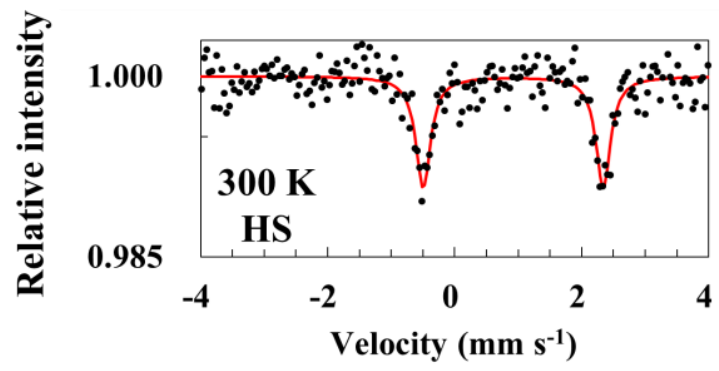

(c)

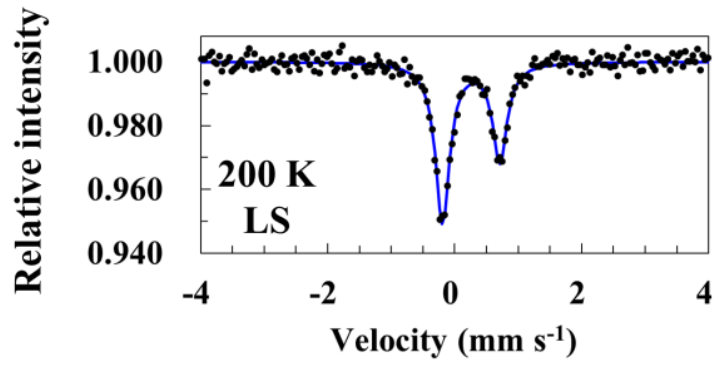

Figure S3. (a) $\chi_{\mathrm{m}} T-T$ plot of [10Me]. Mössbauer spectra of [10Me] at (b) $300 \mathrm{~K}$ and (c) $200 \mathrm{~K}$.

[10Me] exhibits abrupt spin transition without a thermal hysteresis loop. At temperatures below $200 \mathrm{~K}$, the $\chi_{\mathrm{m}} T$ value reflects the LS nature of the Fe(II) complex. The $\chi_{\mathrm{m}} T$ value abruptly increased from $0.05 \mathrm{~cm}^{3} \mathrm{~K} \mathrm{~mol}^{-1}$ at $210 \mathrm{~K}$ to $3.31 \mathrm{~cm}^{3} \mathrm{~K} \mathrm{~mol}^{-1}$ at $350 \mathrm{~K}$, which can be associated with a HS Fe(II) complex. The Mössbauer spectra parameters of [10Me] at 300 and $200 \mathrm{~K}$ are listed in Table S1. The spectrum at $300 \mathrm{~K}$ displays a single quadrupole doublet with IS and QS values of $0.922(8)$ and $2.835(16) \mathrm{mm} \mathrm{s}^{-1}$, respectively. We assigned this component as the Fe(II) HS isomer. The spectrum at $200 \mathrm{~K}$ shows a single asymmetric quadrupole doublet with an IS and QS of 0.231(3) and 0.903(6) $\mathrm{mm} \mathrm{s}^{-1}$, respectively. The intensity ratio of the left (low velocity) and right (high velocity) peaks is 1.624 , where the asymmetric profile is derived from the preferred orientation of the sample having the characteristics of a thin, plate-like crystal habit (we checked for any change in the intensity ratio between a ground sample and an as-grown crystalline sample). We assigned this component as the LS Fe(II) isomer. These results confirm that the change in the magnetic properties of [10Me] originates from the spin transition. 
(a)

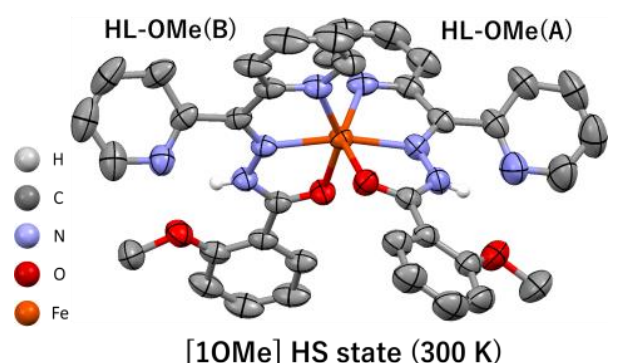

(b)

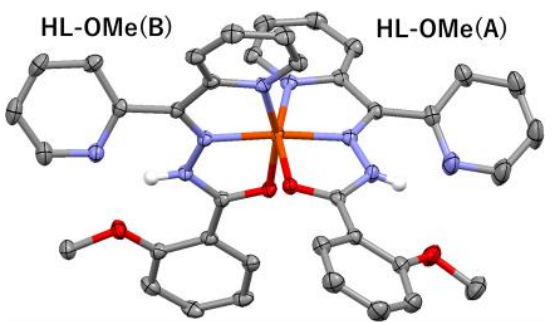

[10Me] LS state $(150 \mathrm{~K})$

(e) (A)

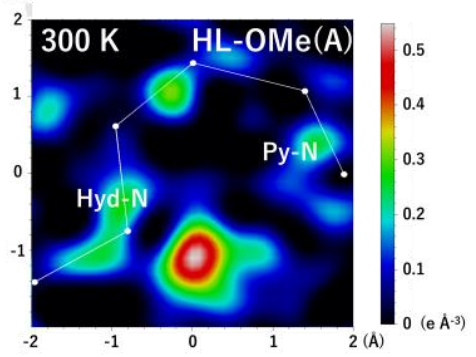

(g) (A)

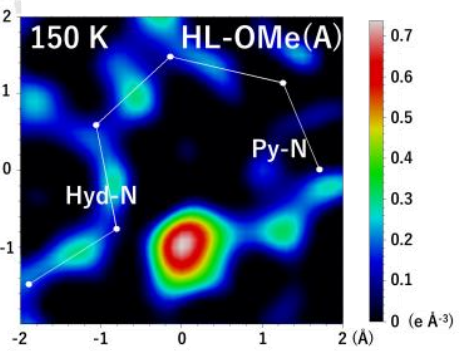

(c) $\mathrm{HS}[10 \mathrm{Me}]_{\mathrm{Hyd}}$

$\mathrm{HL}-\mathrm{OMe}(\mathrm{B}) \rightarrow \mathrm{HL}-\mathrm{OMe}(\mathrm{A})$

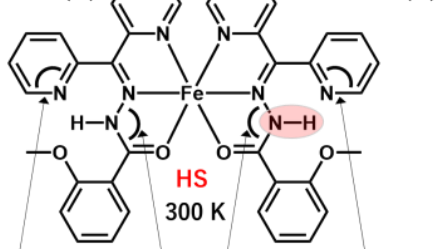

$117.6^{\circ} 114.4^{\circ} 114.4^{\circ} 117.7^{\circ}$

(d)

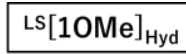

$\mathrm{HL}-\mathrm{OMe}(\mathrm{B}) \longrightarrow \mathrm{HL}-\mathrm{OMe}(\mathrm{A}$

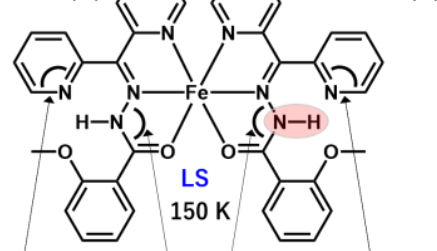

$117.3^{\circ} 113.2^{\circ} 112.8^{\circ} 118.7^{\circ}$

(f) $\frac{(A)}{2}$

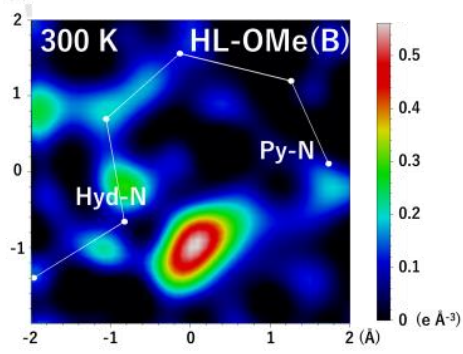

(h) ${ }_{2}^{(A)}$

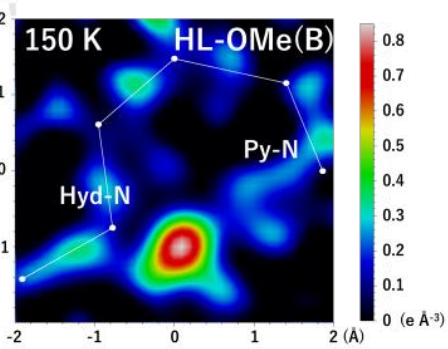

Figure S4. Crystal structure of [10Me] at (a) $300 \mathrm{~K}$ and (b) $150 \mathrm{~K}$ showing $50 \%$ probability displacement ellipsoids. Hydrogen atoms on carbon atoms are omitted for clarity. Schematic of the molecular structure of [10Me] at (c) $300 \mathrm{~K}$ and (d) $150 \mathrm{~K}$. The binding of the two ligands to the Fe(II) ion can be distinguished from the distance between Hyd-N and Py-N. At $150 \mathrm{~K}$, the distance between Hyd-N and Py-N in one of the two ligands was 2.637(3) $\AA$, whereas that in the other ligand was 2.745(3) $\AA$. The ligands with relatively short and long $\mathrm{N} \cdots \mathrm{N}$ distances are denoted as HL-OMe(A) and HL-OMe(B), respectively. Difference Fourier maps of [10Me] around the intramolecular hydrogen bond in (e) HL-OMe(A) at $300 \mathrm{~K}$, (f) $\mathrm{HL}-\mathrm{OMe}(\mathrm{B})$ at $300 \mathrm{~K}$, (g) HL-OMe(A) at $150 \mathrm{~K}$, and $(\mathrm{h}) \mathrm{HL}-\mathrm{OMe}(\mathrm{B})$ at $150 \mathrm{~K}$. The standard deviations of the electron density in the difference Fourier maps at 300 and $150 \mathrm{~K}$ were 0.096 and 0.055 , respectively. The maximum electron density at the expected position of the $\mathrm{H}$ atom was $0.52 \mathrm{e} \AA^{-3}$ in $\mathrm{HL}-\mathrm{OMe}(\mathrm{A})$ at $300 \mathrm{~K}, 0.50 \mathrm{e}^{-3}$ in $\mathrm{HL}-\mathrm{OMe}(\mathrm{B})$ at $300 \mathrm{~K}, 0.68 \mathrm{e}^{-3}$ in $\mathrm{HL}-\mathrm{OMe}(\mathrm{A})$ at $150 \mathrm{~K}$, and $0.73 \mathrm{e} \AA^{-3}$ in $\mathrm{HL}-\mathrm{OMe}(\mathrm{B})$ at $150 \mathrm{~K}$.

The molecular arrangement and anion location of [10Me] were similar to those of [1Cl]. The atoms within the Fe(II) complexes in [10Me] are labeled in the same manner as those in [1Cl], except for the chloro moieties (Fig. S1). The corresponding crystallographic parameters and the coordination distances and angles are listed in Tables S2 and S3, respectively. The metal-toligand bond lengths between Fe(II) and azomethine-N (N2-Fe1) at 150 and $300 \mathrm{~K}$ were typical of the LS (1.858(2) A) and HS (2.097(2) $\AA$ ) states, respectively. These results revealed the spin transition behavior of [10Me]. The proton positions in [10Me] were assigned on the basis of the $\mathrm{NN}_{\mathrm{Hyd}} \mathrm{C}$ and $\mathrm{CN}_{\mathrm{Py}} \mathrm{C}$ angles. The $\mathrm{NN}_{\mathrm{Hyd}} \mathrm{C}$ and $\mathrm{CN}_{\mathrm{Py}} \mathrm{C}$ angles in HL-OMe (A) and $\mathrm{HL}-\mathrm{OMe}(\mathrm{B})$ at 150 and $300 \mathrm{~K}$ were in the ranges $112.8-114.4^{\circ}$ and $117.3-118.7^{\circ}$, respectively. These values demonstrated that, with the decrease in temperature from 300 to $150 \mathrm{~K}$, the protons in $\mathrm{HL}-\mathrm{OMe}(\mathrm{A})$ and $\mathrm{HL}-\mathrm{OMe}(\mathrm{B})$ of [10Me] remain located at Hyd-N despite the change in the spin state from HS to LS. The $\mathrm{H}$ atom positions in HL-OMe(A) and HL-OMe(B) at 150 and $300 \mathrm{~K}$ were determined from the difference Fourier maps. Furthermore, the $\mathrm{CN}_{\mathrm{Py}} \mathrm{C}$ and $\mathrm{NN}_{\mathrm{Hyd}} \mathrm{C}$ angles obtained in the DFT calculation study of [10Me] supported the absence of proton transfer in [10Me] (Table S5). Thus, in contrast to [1Cl], [10Me] exhibited a spin transition without proton transfer between $\mathrm{Hyd}-\mathrm{N}$ and $\mathrm{Py}-\mathrm{N}$. 
Table S1. ${ }^{57} \mathrm{Fe}$ Mössbauer spectra parameters for [1Cl] and [1OMe]. IS (isomer shift), QS (quadrupole splitting), LW (line width).

\begin{tabular}{|c|c|c|c|c|c|c|c|c|}
\hline & \multicolumn{4}{|c|}{ Low spin state } & \multicolumn{4}{|c|}{ High spin state } \\
\hline & \multicolumn{8}{|c|}{$[1 \mathrm{Cl}]$} \\
\hline $\begin{array}{c}\mathrm{T} \\
(\mathrm{K})\end{array}$ & $\begin{array}{c}\text { Fraction } \\
(\%)\end{array}$ & $\begin{array}{c}\text { IS } \\
\left(\mathrm{mm} \mathrm{s}^{-1}\right)\end{array}$ & $\begin{array}{c}\mathrm{QS} \\
\left(\mathrm{mm} \mathrm{s}^{-1}\right)\end{array}$ & $\begin{array}{c}\mathrm{LW} \\
\left(\mathrm{mm} \mathrm{s}^{-1}\right)\end{array}$ & $\begin{array}{c}\text { Fraction } \\
(\%)\end{array}$ & $\begin{array}{c}\text { IS } \\
\left(\mathrm{mm} \mathrm{s}^{-1}\right)\end{array}$ & $\begin{array}{c}\mathrm{QS} \\
\left(\mathrm{mm} \mathrm{s}^{-1}\right)\end{array}$ & $\begin{array}{c}\mathrm{LW} \\
\left(\mathrm{mm} \mathrm{s}^{-1}\right)\end{array}$ \\
\hline 300 & 14 & $0.10(7)$ & $0.99(15)$ & $0.34(19)$ & 86 & $0.792(7)$ & $2.544(14)$ & $0.23(2)$ \\
\hline \multirow[t]{2}{*}{60} & 100 & $0.292(8)$ & $0.913(14)$ & $0.46(9)$ & 0 & - & - & - \\
\hline & \multicolumn{8}{|c|}{ [1OMe] } \\
\hline 300 & 0 & - & - & - & 100 & $0.922(8)$ & $2.835(16)$ & $0.28(2)$ \\
\hline 200 & 100 & $0.231(3)$ & $0.903(6)$ & $0.277(7)$ & 0 & - & - & - \\
\hline
\end{tabular}


Table S2. Crystallographic parameters for crystal structures of [1Cl] at $180 \mathrm{~K}, 350 \mathrm{~K}$, and before and after irradiation with $532 \mathrm{~nm}$ light at $25 \mathrm{~K}$ and [10Me] at $150 \mathrm{~K}$ and $300 \mathrm{~K}$.

\begin{tabular}{|c|c|c|c|c|c|c|}
\hline & \multicolumn{4}{|c|}{$[1 \mathrm{Cl}]$} & \multicolumn{2}{|c|}{ [10Me] } \\
\hline Data & LT phase & HT phase & Before irradiation & After irradiation & LT phase & HT phase \\
\hline Temperature (K) & 180 & 350 & 25 & 25 & 150 & 300 \\
\hline Empirical formula & $\mathrm{C}_{36} \mathrm{H}_{26} \mathrm{As}_{2}$ & $\mathrm{C}_{36} \mathrm{H}_{26} \mathrm{As}_{2}$ & $\mathrm{C}_{36} \mathrm{H}_{26} \mathrm{As}_{2}$ & $\mathrm{C}_{36} \mathrm{H}_{26} \mathrm{As}_{2}$ & $\mathrm{C}_{38} \mathrm{H}_{32} \mathrm{Cl}_{2}$ & $\mathrm{C}_{38} \mathrm{H}_{32} \mathrm{Cl}_{2}$ \\
\hline 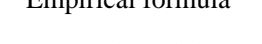 & $\mathrm{F}_{12} \mathrm{Cl}_{2} \mathrm{FeN}_{8} \mathrm{O}_{2}$ & $\mathrm{~F}_{12} \mathrm{Cl}_{2} \mathrm{FeN}_{8} \mathrm{O}_{2}$ & $\mathrm{~F}_{12} \mathrm{Cl}_{2} \mathrm{FeN}_{8} \mathrm{O}_{2}$ & $\mathrm{~F}_{12} \mathrm{Cl}_{2} \mathrm{FeN}_{8} \mathrm{O}_{2}$ & $\mathrm{FeN}_{8} \mathrm{O}_{12}$ & $\mathrm{FeN}_{8} \mathrm{O}_{12}$ \\
\hline Formula weight & 1107.24 & 1107.24 & 1107.24 & 1107.24 & 919.46 & 919.46 \\
\hline Crystal system & monoclinic & monoclinic & monoclinic & monoclinic & monoclinic & monoclinic \\
\hline Space group & $\mathrm{P} 2{ }_{1} / \mathrm{n}$ & $\mathrm{P} 2_{1} / \mathrm{n}$ & $\mathrm{P} 2_{1} / \mathrm{n}$ & $\mathrm{P} 2_{1} / \mathrm{n}$ & $\mathrm{P} 2_{1} / \mathrm{n}$ & $\mathrm{P} 2_{1} / \mathrm{n}$ \\
\hline $\mathrm{a} / \AA ̊$ & $16.5677(3)$ & $16.8760(8)$ & $16.4896(6)$ & $16.8192(11)$ & $16.3409(8)$ & $16.5756(9)$ \\
\hline $\mathrm{b} / \mathrm{A}$ & $16.0511(2)$ & $16.1720(5)$ & $16.0053(4)$ & $15.8059(8)$ & $15.5665(5)$ & $15.8984(7)$ \\
\hline $\mathrm{c} / \AA ̊$ & $16.6587(3)$ & $17.2329(8)$ & 16.6301(6) & $17.0759(11)$ & $16.8955(8)$ & $17.27953(10)$ \\
\hline$\alpha /{ }^{\circ}$ & 90 & 90 & 90 & 90 & 90 & 90 \\
\hline$\beta /{ }^{\circ}$ & $116.445(2)$ & 117.797(6) & $116.615(3)$ & $117.852(8)$ & 117.187(6) & 118.081(7) \\
\hline$\gamma /{ }^{\circ}$ & 90 & 90 & 90 & 90 & 90 & 90 \\
\hline Volume $/ \AA^{3}$ & $3966.49(13)$ & $4160.5(4)$ & $3924.0(3)$ & 4013.6(5) & $3822.9(3)$ & 4021.2(4) \\
\hline $\mathrm{Z}$ & 4 & 4 & 4 & 4 & 4 & 4 \\
\hline$\rho_{\text {calc }} \mathrm{g} / \mathrm{cm}^{3}$ & 1.854 & 1.768 & 1.874 & 1.832 & 1.598 & 1.519 \\
\hline$\mu / \mathrm{mm}^{-1}$ & 2.275 & 2.169 & 2.299 & 2.248 & 0.612 & 0.582 \\
\hline$F(000)$ & 2192.0 & 2192.0 & 2192.0 & 2192.0 & 1888.0 & 1888.0 \\
\hline Radiation & $\operatorname{MoK} \alpha$ & $\mathrm{MoK} \alpha$ & $\mathrm{MoK} \alpha$ & $\mathrm{MoK} \alpha$ & $\mathrm{MoK} \alpha$ & $\mathrm{MoK} \alpha$ \\
\hline & $(\lambda=0.71073)$ & $(\lambda=0.71073)$ & $(\lambda=0.71073)$ & $(\lambda=0.71073)$ & $(\lambda=0.71073)$ & $(\lambda=0.71073)$ \\
\hline $\begin{array}{l}2 \Theta \text { range for data } \\
\text { collection } /{ }^{\circ}\end{array}$ & 3.728 to 52.744 & 3.672 to 52.744 & 4.682 to 52.742 & 5.154 to 50.698 & 3.834 to 61.43 & 4.678 to 61.362 \\
\hline Index ranges & $\begin{aligned}-20 & \leq \mathrm{h} \leq 20 \\
-20 & \leq \mathrm{k} \leq 20 \\
-20 & \leq 1 \leq 20\end{aligned}$ & $\begin{array}{l}-21 \leq \mathrm{h} \leq 21 \\
-20 \leq \mathrm{k} \leq 20 \\
-21 \leq 1 \leq 20\end{array}$ & $\begin{aligned}-20 & \leq \mathrm{h} \leq 20 \\
-18 & \leq \mathrm{k} \leq 20 \\
-20 & \leq 1 \leq 20\end{aligned}$ & $\begin{array}{r}-19 \leq \mathrm{h} \leq 20 \\
-19 \leq \mathrm{k} \leq 19 \\
-20 \leq 1 \leq 19\end{array}$ & $\begin{array}{l}-23 \leq \mathrm{h} \leq 22 \\
-21 \leq \mathrm{k} \leq 22 \\
-24 \leq 1 \leq 24\end{array}$ & $\begin{array}{l}-21 \leq \mathrm{h} \leq 23 \\
-22 \leq \mathrm{k} \leq 17 \\
-21 \leq 1 \leq 24\end{array}$ \\
\hline $\begin{array}{l}\text { Reflections } \\
\text { collected }\end{array}$ & 61932 & 33470 & 38574 & 34920 & 33311 & 35853 \\
\hline $\begin{array}{l}\text { Independent } \\
\text { reflections }\end{array}$ & $\begin{array}{c}8088 \\
{\left[R_{\text {int }}=0.0330,\right.} \\
\left.\mathrm{R}_{\text {sigma }}=0.0181\right]\end{array}$ & $\begin{array}{c}8479 \\
{\left[R_{\text {int }}=0.0544\right.} \\
\left.R_{\text {sigma }}=0.0565\right]\end{array}$ & $\begin{array}{c}8021 \\
{\left[R_{\text {int }}=0.0660,\right.} \\
\left.R_{\text {sigma }}=0.0500\right]\end{array}$ & $\begin{array}{c}7345 \\
{\left[\mathrm{R}_{\text {int }}=0.0866,\right.} \\
\left.\mathrm{R}_{\text {sigma }}=0.0748\right]\end{array}$ & $\begin{array}{c}10279 \\
{\left[\mathrm{R}_{\text {int }}=0.0579\right.} \\
\left.\mathrm{R}_{\text {sigma }}=0.0610\right]\end{array}$ & $\begin{array}{c}10813 \\
{\left[\mathrm{R}_{\text {int }}=0.0232,\right.} \\
\left.\mathrm{R}_{\text {sigma }}=0.0294\right]\end{array}$ \\
\hline $\begin{array}{c}\text { Data/restraints } \\
\text { /parameters }\end{array}$ & $8088 / 0 / 576$ & $8479 / 0 / 576$ & $8021 / 0 / 568$ & $7345 / 336 / 632$ & $10279 / 0 / 560$ & $10813 / 8 / 634$ \\
\hline Goodness-of-fit on $\mathrm{F}^{2}$ & 1.025 & 1.046 & 1.062 & 1.037 & 1.069 & 1.055 \\
\hline $\begin{array}{l}\text { Final } R \text { indexes } \\
\quad[\mathrm{I}>=2 \sigma(\mathrm{I})]\end{array}$ & $\begin{array}{l}\mathrm{R}_{1}=0.0297 \\
\mathrm{wR}_{2}=0.0700\end{array}$ & $\begin{array}{c}\mathrm{R}_{1}=0.0702, \\
\mathrm{wR}_{2}=0.1840\end{array}$ & $\begin{array}{l}\mathrm{R}_{1}=0.0465, \\
\mathrm{wR}_{2}=0.1011\end{array}$ & $\begin{array}{l}\mathrm{R}_{1}=0.0655, \\
\mathrm{wR}_{2}=0.1285\end{array}$ & $\begin{aligned} \mathrm{R}_{1} & =0.0524, \\
\mathrm{wR}_{2} & =0.1354\end{aligned}$ & $\begin{array}{l}\mathrm{R}_{1}=0.0445, \\
\mathrm{wR}_{2}=0.1173\end{array}$ \\
\hline $\begin{array}{c}\text { Final } \mathrm{R} \text { indexes } \\
\text { [all data] }\end{array}$ & $\begin{array}{c}\mathrm{R}_{1}=0.0353, \\
\mathrm{wR}_{2}=0.0728\end{array}$ & $\begin{array}{l}\mathrm{R}_{1}=0.1279, \\
\mathrm{wR}_{2}=0.2157\end{array}$ & $\begin{array}{l}\mathrm{R}_{1}=0.0597, \\
\mathrm{wR}_{2}=0.1086\end{array}$ & $\begin{array}{c}\mathrm{R}_{1}=0.1062, \\
\mathrm{wR}_{2}=0.1489\end{array}$ & $\begin{array}{c}\mathrm{R}_{1}=0.0688 \\
\mathrm{wR}_{2}=0.1438\end{array}$ & $\begin{array}{c}\mathrm{R}_{1}=0.0733, \\
\mathrm{wR}_{2}=0.1289\end{array}$ \\
\hline
\end{tabular}


Table S3. Coordination distances, angles, and intramolecular hydrogen bonding parameters in [1Cl] at $180 \mathrm{~K}, 350 \mathrm{~K}$, and before and after irradiation with $532 \mathrm{~nm}$ light at $25 \mathrm{~K}$, and [10Me] at 150 and $300 \mathrm{~K}$. The labels corresponding to the distances and angles in $\mathrm{HL}-\mathrm{Cl}(\mathrm{A})$ and $\mathrm{HL}-\mathrm{OMe}(\mathrm{A})$ are marked with $(*)$ for clarity.

\begin{tabular}{|c|c|c|c|c|c|c|}
\hline & \multicolumn{4}{|c|}{$[1 \mathrm{Cl}]$} & \multicolumn{2}{|c|}{ [10Me] } \\
\hline Data & LT phase & HT phase & $\begin{array}{c}\text { Before } \\
\text { irradiation }\end{array}$ & $\begin{array}{c}\text { After } \\
\text { irradiation }\end{array}$ & $\begin{array}{c}\text { LT } \\
\text { phase }\end{array}$ & $\begin{array}{c}\text { HT } \\
\text { phase }\end{array}$ \\
\hline $\begin{array}{c}\text { Temperature } \\
(\mathrm{K})\end{array}$ & 180 & 350 & 25 & 25 & 150 & 300 \\
\hline Spin State & LS & HS & LS & HS & LS & HS \\
\hline \multicolumn{7}{|c|}{ Coordination distances around $\mathrm{Fe}(\mathrm{II})(\AA)$} \\
\hline$* \mathrm{Fe} 1-\mathrm{N} 1$ & $1.958(2)$ & $2.158(5)$ & $1.975(3)$ & $2.163(5)$ & $1.950(2)$ & $2.158(2)$ \\
\hline$* \mathrm{Fe} 1-\mathrm{N} 2$ & $1.851(2)$ & $2.111(6)$ & $1.872(4)$ & $2.119(6)$ & $1.858(2)$ & $2.097(2)$ \\
\hline *Fe1-O1 & $1.955(2)$ & $2.121(4)$ & $1.976(3)$ & $2.125(5)$ & $1.971(2)$ & $2.115(1)$ \\
\hline $\mathrm{Fe} 1-\mathrm{N} 5$ & $1.954(1)$ & $2.145(4)$ & $1.965(2)$ & $2.138(5)$ & $1.937(2)$ & 2.104(1) \\
\hline Fe1-N6 & $1.870(2)$ & $2.122(5)$ & $1.893(4)$ & $2.114(6)$ & $1.870(2)$ & $2.118(2)$ \\
\hline $\mathrm{Fe} 1-\mathrm{O} 2$ & $1.976(1)$ & $2.106(3)$ & $1.991(2)$ & $2.097(4)$ & $1.958(1)$ & 2.081(1) \\
\hline \multicolumn{7}{|c|}{$\mathrm{CN}_{\mathrm{Py}} \mathrm{C}$ angle $\left(^{\circ}\right)$} \\
\hline *C7-N4-C11 & $123.5(2)$ & $118.0(7)$ & $123.4(4)$ & $118.4(7)$ & $118.7(3)$ & $117.7(3)$ \\
\hline $\mathrm{C} 25-\mathrm{N} 8-\mathrm{C} 29$ & $118.0(2)$ & $117.3(6)$ & $117.7(4)$ & $117.9(7)$ & $117.3(2)$ & $117.6(3)$ \\
\hline \multicolumn{7}{|c|}{$\mathrm{NN}_{\mathrm{Hyd}} \mathrm{C}$ angle $\left({ }^{\circ}\right)$} \\
\hline *N2-N3-C12 & $108.1(2)$ & $114.5(5)$ & $108.1(3)$ & $114.2(5)$ & $112.8(2)$ & $114.4(2)$ \\
\hline N6-N7-C30 & $113.2(2)$ & $115.0(5)$ & $113.2(3)$ & $113.6(5)$ & $113.2(2)$ & $114.4(2)$ \\
\hline \multicolumn{7}{|c|}{ Intramolecular hydrogen bond parameter $(\AA)$} \\
\hline$* \mathrm{~N} 3 \cdot \cdot \mathrm{N} 4$ & $2.610(3)$ & $2.630(9)$ & $2.599(5)$ & $2.633(9)$ & $2.637(3)$ & $2.684(3)$ \\
\hline$* \mathrm{~N} 3 \cdot \cdot \mathrm{H} 1$ & $1.97(3)$ & $0.91(7)$ & - & - & $0.76(4)$ & $0.77(3)$ \\
\hline$* \mathrm{~N} 4 \cdot \cdot \mathrm{H} 1$ & $0.84(3)$ & $1.96(7)$ & - & - & $2.08(3)$ & $2.11(3)$ \\
\hline $\mathrm{N} 7 \cdot \cdot \mathrm{N} 8$ & $2.651(3)$ & $2.693(8)$ & $2.646(5)$ & $2.673(9)$ & $2.745(3)$ & $2.798(3)$ \\
\hline $\mathrm{N} 7 \cdot \cdot \mathrm{H} 2$ & $0.81(3)$ & $0.85(6)$ & - & - & $0.88(4)$ & $0.78(3)$ \\
\hline $\mathrm{N} 8 \cdot \cdot \mathrm{H} 2$ & 2.04(3) & $2.10(5)$ & - & - & 2.11(3) & $2.27(2)$ \\
\hline
\end{tabular}


Table S4. List of the $\mathrm{NN}_{\mathrm{Hyd}} \mathrm{C}$ angles centered on the protonated or deprotonated nitrogen atoms of the hydrazone moiety in the reported transition-metal complexes coordinated with hydrazone ligands.

\begin{tabular}{|c|c|c|c|c|c|}
\hline Ref & $\mathrm{R} 1$ & $\mathrm{R} 2$ & $\begin{array}{c}\text { Metal, } \\
\text { valence, spin state }\end{array}$ & $\begin{array}{c}\text { Protonated or } \\
\text { non-protonated }\end{array}$ & $\begin{array}{c}\mathrm{NN}_{\mathrm{Hy}} \mathrm{C} \text { angle centered on } \\
\text { Hydrazone-N }\end{array}$ \\
\hline \multirow{2}{*}{1} & \multirow{2}{*}{ Phenyl } & \multirow{2}{*}{ Phenyl } & \multirow{2}{*}{$\mathrm{Ni}(\mathrm{II})$} & Protonated & $113.8(2)$ \\
\hline & & & & Non-Protonated & $108.9(2)$ \\
\hline \multirow{4}{*}{2} & \multirow{2}{*}{ Phenyl } & \multirow{2}{*}{ Phenyl } & \multirow{2}{*}{$\mathrm{Ni}(\mathrm{II})$} & Protonated & $113.3(3)$ \\
\hline & & & & Non-Protonated & $108.9(3)$ \\
\hline & \multirow{2}{*}{ Phenyl } & \multirow{2}{*}{ Phenyl } & \multirow{2}{*}{$\mathrm{Ni}(\mathrm{II})$} & Non-Protonated & $107.5(1)$ \\
\hline & & & & Non-Protonated & $107.7(1)$ \\
\hline \multirow{4}{*}{3} & \multirow{2}{*}{ Phenyl } & \multirow{2}{*}{ Phenyl } & \multirow{2}{*}{$\mathrm{Ni}(\mathrm{II})$} & Non-Protonated & $108.8(5)$ \\
\hline & & & & Non-Protonated & $108.7(4)$ \\
\hline & \multirow{2}{*}{ Phenyl } & \multirow{2}{*}{ Phenyl } & $7 \mathrm{n}(\mathrm{II})$ & Non-Protonated & $107.7(3)$ \\
\hline & & & $\operatorname{Ln}(11)$ & Non-Protonated & $107.5(3)$ \\
\hline & & & & Non-Protonated & $107.1(8)$ \\
\hline 4 & Phenyl & 4-Py & Fe(II)-LS & Non-Protonated & $106.8(8)$ \\
\hline 4 & & & & Non-Protonated & $106.9(6)$ \\
\hline & Phenyl & Phenyl & $\mathrm{Fe}(\mathrm{II})$-LS & Non-Protonated & $107.0(6)$ \\
\hline & & & & Non-Protonated & $108.2(2)$ \\
\hline 5 & Phenyl & Phenyl & $\mathrm{Zn}(\mathrm{II})$ & Non-Protonated & $108.1(2)$ \\
\hline 5 & Phenyl & $4-\mathrm{Cl}$ & $\mathrm{Zn}(\mathrm{II})$ & Protonated & $115.6(3)$ \\
\hline & Phenyl & $4-\mathrm{NO}_{2}$ & $\mathrm{Zn}(\mathrm{II})$ & Non-Protonated & $108.1(7)$ \\
\hline & & & & Non-Protonated & $109.9(2)$ \\
\hline & Phenyl & $2-\mathrm{NH}_{2}$ & $\operatorname{Ln}(\mathrm{II})$ & Non-Protonated & $109.3(2)$ \\
\hline & & & & Non-Protonated & $109.0(4)$ \\
\hline 6 & Phenyl & $2-\mathrm{NH}_{2}$ & N1(II) & Non-Protonated & $108.7(4)$ \\
\hline & & & & Non-Protonated & $109.6(4)$ \\
\hline & Phenyl & $2-\mathrm{NH}_{2}$ & $\operatorname{Mn}(\mathrm{II})-\mathrm{HS}$ & Non-Protonated & $109.6(3)$ \\
\hline & Phenyl & 4-Py & $\mathrm{Mn}(\mathrm{II})-\mathrm{HS}$ & Protonated & $114.0(1)$ \\
\hline 7 & Phenyl & 4-Py & Mn(II)-HS & Non-Protonated & $109.3(2)$ \\
\hline & Phenyl & 4-Py & $\mathrm{Mn}(\mathrm{II})-\mathrm{HS}$ & Non-Protonated & $108.54(9)$ \\
\hline 8 & Phenyl & 4-Py & $\mathrm{Zn}(\mathrm{II})$ & Non-Protonated & $108.0(2)$ \\
\hline 8 & Phenyl & 4-Py & $\mathrm{Zn}(\mathrm{II})$ & Non-Protonated & $108.2(2)$ \\
\hline & Phenyl & 4-Py & $\mathrm{Zn}(\mathrm{II})$ & Non-Protonated & $108.9(2)$ \\
\hline 0 & Phenyl & 4-Py & $\mathrm{Zn}(\mathrm{II})$ & Non-Protonated & $107.9(4)$ \\
\hline 9 & Phenyl & 4-Py & $\mathrm{Zn}(\mathrm{II})$ & Non-Protonated & $107.6(2)$ \\
\hline & Phenyl & 4-Py & $\mathrm{Zn}(\mathrm{II})$ & Non-Protonated & $108.3(1)$ \\
\hline & & & & Non-Protonated & $108.2(1)$ \\
\hline & $\mathrm{H}$ & Phenyl & $\mathrm{Fe}(\mathrm{II})-\mathrm{HS}$ & Non-Protonated & $107.4(1)$ \\
\hline & Me & Phenvl & $\mathrm{Fe}(\mathrm{II})-\mathrm{I} \mathrm{S}$ & Non-Protonated & $107.6(8)$ \\
\hline & IMe & Phenyı & Fe(II)-LS & Non-Protonated & $106.7(8)$ \\
\hline & & & & Non-Protonated & $106.8(2)$ \\
\hline 10 & Phenyl & Phenyl & Fe(II)-LS & Non-Protonated & $106.8(2)$ \\
\hline 10 & $\mathrm{H}$ & 2 & $\mathrm{Fe}(\mathrm{II})-\mathrm{HS}$ & Non-Protonated & $109.7(3)$ \\
\hline & П & L-Uत & Fe(11)-Hs & Non-Protonated & $109.6(3)$ \\
\hline & $\mathrm{Me}$ & $2-\mathrm{OH}$ & $\mathrm{Fe}(\mathrm{II})-\mathrm{LS}$ & Non-Protonated & $108.8(2)$ \\
\hline & $\mathrm{Me}$ & $2-\mathrm{OH}$ & $\mathrm{Fe}(\mathrm{II})-\mathrm{HS}$ & Non-Protonated & $109.4(4)$ \\
\hline & Phenvl & $2-\mathrm{OH}$ & $\mathrm{Fe}(\mathrm{II})-\mathrm{I} \mathrm{S}$ & Non-Protonated & $108.2(2)$ \\
\hline & Pnenyı & $2-O H$ & Fe(II)-LS & Non-Protonated & $108.1(2)$ \\
\hline & & & & Non-Protonated & $108.6(3)$ \\
\hline & $\mathrm{Me}$ & Phenyl & Mn(II)-HS & Non-Protonated & $107.6(3)$ \\
\hline 11 & $\mathrm{H}$ & Phenvl & $\mathrm{Zn}(\mathrm{II})$ & Non-Protonated & 107.6(2), \\
\hline & & & & Non-Protonated & $109.2(2)$ \\
\hline & $\mathrm{Me}$ & Phenyl & $\mathrm{Zn}(\mathrm{II})$ & Non-Protonated & 109.14(9) \\
\hline 12 & & & & Non-Protonated & $108.2(3)$ \\
\hline 12 & Phenyl & 4-Py & $\operatorname{Ln}(\mathrm{II})$ & Protonated & $114.4(3)$ \\
\hline & $\mathrm{H}$ & Phenyl & $\mathrm{Ni}(\mathrm{II})$ & Protonated & $114.7(2)$ \\
\hline & $\mathrm{H}$ & Phenvl & $\mathrm{Ni}(\mathrm{II})$ & Non-Protonated & $108.7(1)$ \\
\hline 13 & H & Pnenyı & N1(II) & Non-Protonated & $108.2(1)$ \\
\hline & $\mathrm{H}$ & Phenyl & $\mathrm{Ni}(\mathrm{II})$ & Protonated & $114.8(2)$ \\
\hline & $\mathrm{H}$ & Phenyl & $\mathrm{Ni}(\mathrm{II})$ & Non-Protonated & 109.1(4) \\
\hline
\end{tabular}




\begin{tabular}{|c|c|c|c|c|c|}
\hline & & & & Protonated & $114.0(3)$ \\
\hline \multirow{2}{*}{14} & \multirow{2}{*}{$\mathrm{H}$} & \multirow{2}{*}{ Phenyl } & \multirow{2}{*}{$\mathrm{Mn}(\mathrm{II})-\mathrm{HS}$} & Non-Protonated & $108.9(1)$ \\
\hline & & & & Non-Protonated & 107.8(1) \\
\hline 15 & $\mathrm{H}$ & Phenyl & $\mathrm{Zn}(\mathrm{II})$ & Protonated & 116.6(1) \\
\hline 16 & $\mathrm{H}$ & Phenyl & $\mathrm{Ni}(\mathrm{II})$ & Non-Protonated & $108.0(2)$ \\
\hline \multirow[b]{2}{*}{17} & \multirow{2}{*}{$\mathrm{H}$} & \multirow[b]{2}{*}{ Phenyl } & \multirow[b]{2}{*}{$\mathrm{Ni}(\mathrm{II})$} & Non-Protonated & $108.5(2)$ \\
\hline & & & & Non-Protonated & $108.2(2)$ \\
\hline \multirow{2}{*}{18} & \multirow{2}{*}{$\mathrm{H}$} & \multirow{2}{*}{ Phenyl } & \multirow{2}{*}{$\mathrm{Mn}(\mathrm{II})-\mathrm{HS}$} & Non-Protonated & $108.7(2)$ \\
\hline & & & & Non-Protonated & 108.1(2) \\
\hline \multirow{2}{*}{19} & $\mathrm{H}$ & $4-\mathrm{Cl}$ & $\mathrm{Zn}(\mathrm{II})$ & Protonated & 114.1(3) \\
\hline & $\mathrm{H}$ & $4-\mathrm{NO}_{2}$ & $\mathrm{Zn}(\mathrm{II})$ & Non-Protonated & $108.3(2)$ \\
\hline 20 & $\mathrm{H}$ & $4-\mathrm{NMe}_{2}$ & $\mathrm{Zn}(\mathrm{II})$ & Protonated & $115.1(2)$ \\
\hline \multirow{4}{*}{21} & \multirow{4}{*}{$\mathrm{H}$} & \multirow{4}{*}{$2-\mathrm{Br}$} & \multirow{4}{*}{ Fe(II)-LS } & Non-Protonated & $107(1)$ \\
\hline & & & & Non-Protonated & $105(1)$ \\
\hline & & & & Non-Protonated & $106(1)$ \\
\hline & & & & Non-Protonated & $106(1)$ \\
\hline 20 & $\mathrm{H}$ & $4-\mathrm{OH}$ & $\mathrm{Fe}(\mathrm{II})-\mathrm{LS}$ & Non-Protonated & $107.8(2)$ \\
\hline 22 & $\mathrm{H}$ & $4-\mathrm{OH}$ & $\mathrm{Fe}(\mathrm{II})-\mathrm{HS}$ & Non-Protonated & $108.5(2)$ \\
\hline & $\mathrm{H}$ & 4-Py & $\mathrm{Zn}(\mathrm{II})$ & Protonated & $114.6(2)$ \\
\hline 23 & 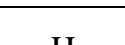 & $4 \mathrm{D}-2>$ & $70(D)>2$ & Non-Protonated & 111.9(2), \\
\hline & $\mathrm{H}$ & 4-Ру & $\operatorname{Ln}(I I)$ & Non-Protonated & 108.7(2) \\
\hline 24 & $\mathrm{Me}$ & 4-Py & Mn(II)-HS & Non-Protonated & 108.2(5) \\
\hline 24 & $\mathrm{Me}$ & 3-Py & $\mathrm{Mn}(\mathrm{II})-\mathrm{HS}$ & Protonated & $114.8(2)$ \\
\hline 25 & $\mathrm{Me}$ & Phenyl & $\mathrm{Mn}(\mathrm{II})-\mathrm{HS}$ & Non-Protonated & $108.7(2)$ \\
\hline 25 & $\mathrm{Me}$ & Phenyl & $\mathrm{Zn}(\mathrm{II})$ & Non-Protonated & 109.3(3) \\
\hline 26 & $\mathrm{Me}$ & Phenyl & $\mathrm{Mn}$ (II)-HS & Non-Protonated & 109.0(1) \\
\hline 27 & $M$ & Dhonul & $\mathrm{Ni}(\mathrm{ID})$ & Non-Protonated & $107.8(5)$ \\
\hline 21 & Mie & Phenyl & $\mathrm{N} 1(I I)$ & Non-Protonated & $109.5(5)$ \\
\hline & Py & 3-Py & $\mathrm{Mn}(\mathrm{II})-\mathrm{HS}$ & Non-Protonated & $109.0(3)$ \\
\hline 20 & Ру & 3-Ру & $\operatorname{Mn}(I I)-H S$ & Non-Protonated & $108.7(3)$ \\
\hline 28 & Phenvl & Phenvl & $\mathrm{Mn}(\mathrm{II})-\mathrm{HS}$ & Non-Protonated & 108.4(1) \\
\hline & Fегіну & Frenyi & ИПП(II)-ПМ & Non-Protonated & 108.4(1) \\
\hline 29 & Py & Phenyl & $\mathrm{Zn}$ (II) & Protonated & $115.8(1)$ \\
\hline & Py & Phenyl & $\mathrm{Mn}(\mathrm{II})-\mathrm{HS}$ & Protonated & $116.0(3)$ \\
\hline & $P_{y}$ & Phenyl & $\mathrm{Mn}$ (II)_HS & Non- Protonated & 108.6(1) \\
\hline 30 & Py & Pnenyl & $\operatorname{lin}(I I)-\mathrm{H} S$ & Non- Protonated & $108.8(1)$ \\
\hline & $\mathrm{P}_{\mathrm{Y}}$ & Phenvl & $\mathrm{Mn}(\mathrm{II}) \mathrm{HS}$ & Non- Protonated & $109.0(3)$ \\
\hline & Py & Phenyl & $\operatorname{Mn}(I I)-H S$ & Non- Protonated & 108.7(3) \\
\hline & $\mathrm{D}$ & $4 \mathrm{D}$ & $N \cdot(D)$ & Non-Protonated & 107.8(3), \\
\hline & Py & 4-Py & $N_{1}(I I)$ & Non-Protonated & 108.8(3) \\
\hline & Py & Phenvl & $\mathrm{Mn}(\mathrm{II})-\mathrm{HS}$ & Non-Protonated & 109.7(7), \\
\hline 31 & $\Gamma_{y}$ & Гпепуг & 1VII(II)-ก & Non-Protonated & $110.4(7)$ \\
\hline 31 & Py & Phenyl & $\mathrm{Ni}(\mathrm{II})$ & Non-Protonated & 108.3(7), \\
\hline & & Phenyl & $N_{1}(I I)$ & Non-Protonated & $109.5(7)$ \\
\hline & $\mathrm{Py}_{\mathrm{y}}$ & Phenyl & $7 n(J)$ & Non-Protonated & 111(1), \\
\hline & Рy & Phenyl & $\operatorname{Ln}(I I)$ & Non-Protonated & $109(1)$ \\
\hline & Py & Phenvl & $\mathrm{Fe}(\mathrm{II})-\mathrm{I}$ S & Non-Protonated & 106.7(5), \\
\hline 32 & ry & Глегіугі & Fе(II)-LS & Non-Protonated & 108.2(5) \\
\hline 32 & Py & $4-\mathrm{Br}$ & $\mathrm{Fe}(\mathrm{II})-\mathrm{I}$ S & Non-Protonated & 107.2(7), \\
\hline & Py & & $\mathrm{Fe}(\mathrm{II})-\mathrm{LS}$ & Non-Protonated & $107.8(7)$ \\
\hline & & & $\mathrm{Fe}(\mathrm{II})-\mathrm{HS}$ & Protonated & $114.5(5)$ \\
\hline$[1 \mathrm{Cl}$ & Py & $2-\mathrm{Cl}$ & Fe(II)-HS & Protonated & $115.0(5)$ \\
\hline [ICI] & Fy & $2-\mathrm{Cl}$ & & Protonated & $113.2(2)$ \\
\hline & & & $\mathrm{Fe}(11)-\mathrm{LS}$ & Non- Protonated & 108.1(2) \\
\hline & & & $\left.\mathrm{E}_{\mathrm{S}} \mathrm{UL}\right) \mathrm{t}$ & Protonated & 114.4(2) \\
\hline 510Me & $\mathrm{P}_{\mathrm{y}}$ & 208 & Ге(II)-ПS & Protonated & 114.4(2) \\
\hline [10ivie] & $r_{y}$ & L-UIVIE & $\mathrm{Fe}(\mathrm{II})-\mathrm{I} \mathrm{S}$ & Protonated & $112.8(2)$ \\
\hline & & & Fe(II)-LS & Protonated & 113.2(2) \\
\hline
\end{tabular}


(a)

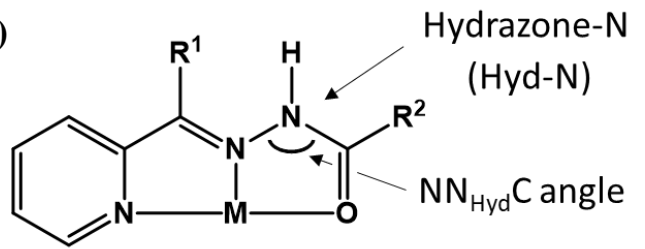

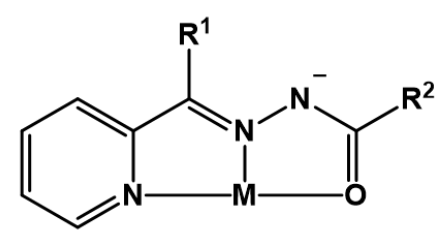

$\mathrm{M}=\mathrm{Mn} ", \mathrm{Fe} ", \mathrm{Ni}{ }^{\prime \prime}$ and $\mathrm{Zn} "$

$\mathrm{R}^{1}=\mathrm{H}$, Methyl, Ethyl, Phenyl, Pyridyl

(b)
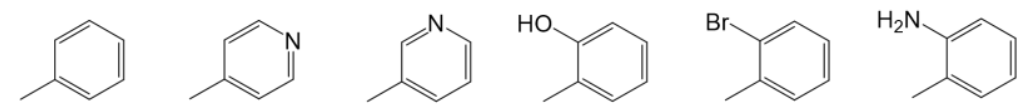

Phenyl

4-Py

3-Py

$2-\mathrm{OH}$

$2-\mathrm{Br}$

2- $\mathrm{NH}_{2}$

$R^{2}=$<smiles>Cc1ccc(N)cc1</smiles>

4- $\mathrm{NH}_{2}$

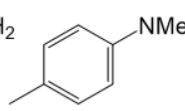

4-NMe 2

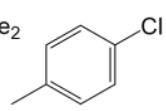

4-Cl

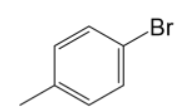

4-Br

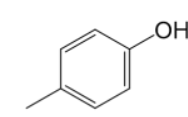

4-OH

(c)

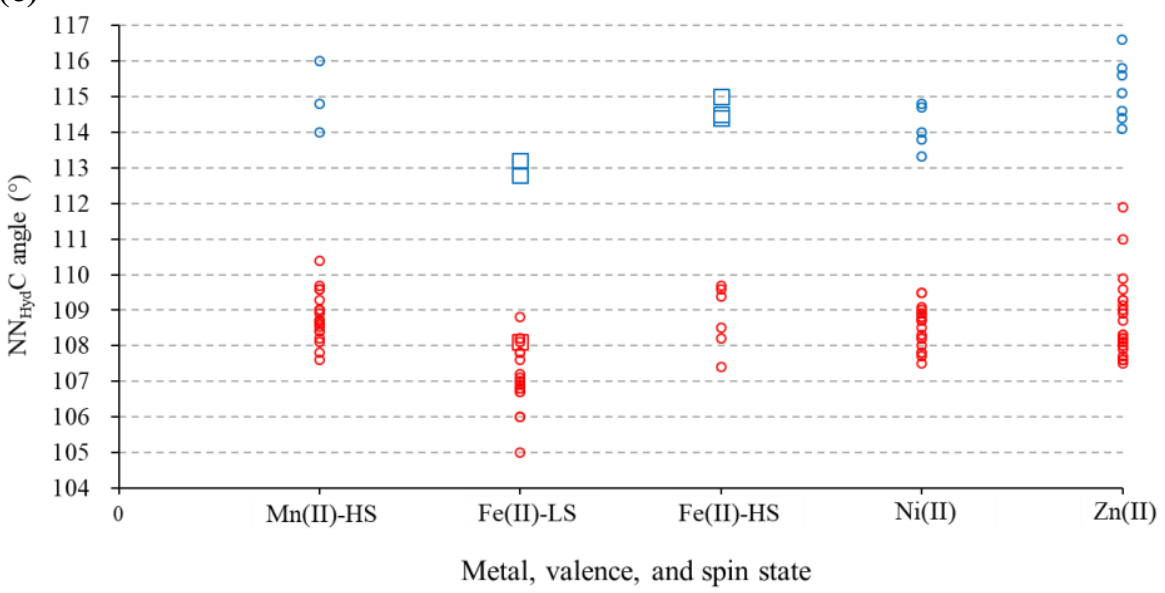

(a) Schematic of a hydrazone complex protonated at Hyd-N (left) and deprotonated at Hyd-N (right). (b) Summary of substituents in hydrazone complexes listed in Table S4. (c) Dependence of the $\mathrm{NN}_{\mathrm{Hyd}} \mathrm{C}$ angle in the protonated or deprotonated states in hydrazone complexes with different metal centers and spin states. Circles and squares represent data collected from reported hydrazone compounds and data collected in this study, respectively. The blue circles and squares are the $\mathrm{NN}_{\mathrm{Hyd}} \mathrm{C}$ angles of the protonated hydrazone complex, and red circles and squares are the $\mathrm{NN}_{\mathrm{Hyd}} \mathrm{C}$ angles of the deprotonated hydrazone complex.

We collected the crystal-structural data of the aforementioned transition-metal (HS Mn(II), HS Fe(II), LS Fe(II), Ni(II), and Zn(II)) complexes bearing hydrazone ligands to explore the influence of protonation or deprotonation on the $\mathrm{NN}_{\mathrm{Hyd}} \mathrm{C}$ angle of the hydrazone moiety (Hyd-N), and the corresponding $\mathrm{NN}_{\mathrm{Hyd}} \mathrm{C}$ angles are listed in Table $\mathrm{S} 4$ and summarized in (c). The $\mathrm{NN}_{\mathrm{Hyd}} \mathrm{C}$ angles increased with protonation at Hyd-N. The ranges of the $\mathrm{NN}_{\mathrm{Hyd}} \mathrm{C}$ angle on the nonprotonated Hyd-N moiety in $\mathrm{HS} \mathrm{Mn}(\mathrm{II}), \mathrm{Ni}(\mathrm{II})$, and $\mathrm{Zn}(\mathrm{II})$ were $108-110^{\circ}, 107-110^{\circ}$, and $107-112^{\circ}$, respectively, whereas the ranges of the $\mathrm{NN}_{\mathrm{Hyd}} \mathrm{C}$ angle at the protonated Hyd- $\mathrm{N}$ moiety in $\mathrm{HS} \mathrm{Mn}(\mathrm{II}), \mathrm{Ni}(\mathrm{II})$, and $\mathrm{Zn}(\mathrm{II})$ were $114-116^{\circ}, 113-115^{\circ}$, and $114-117^{\circ}$, respectively. The range of the $\mathrm{NN}_{\mathrm{Hyd}} \mathrm{C}$ angle at nonprotonated Hyd $-\mathrm{N}$ in previously reported HS Fe(II) hydrazone complexes is $107-110^{\circ}$, which is approximately the same as those of the $\mathrm{HS} \mathrm{Mn}$ (II) and $\mathrm{Ni}$ (II) complexes. However, the values previously reported for the $\mathrm{NN}_{\mathrm{Hyd}} \mathrm{C}$ angle at nonprotonated $\mathrm{Hyd}-\mathrm{N}$ in other LS Fe(II) hydrazone complexes were slightly smaller than that for the HS Fe(II) complex, and the range of the corresponding angle in the LS Fe(II) complex was $105-109^{\circ}$. The corresponding angles in previously reported Fe(II) hydrazone complexes exhibiting a spin transition, [ $\left.\mathrm{Fe}^{\mathrm{II}}(\mathrm{L} 1)_{2}\right]\left(\mathrm{HL} 1=2\right.$-hydroxy- $N^{\prime}$-(1-(pyridin-2-yl)ethylidene)-benzohydrazide) and [ $\left.\mathrm{Fe}^{\mathrm{II}}\left(\mathrm{L}_{2}\right)_{2}\right] \cdot \mathrm{CH}_{3} \mathrm{OH}$ (HL2 = 4-hydroxy- $N^{\prime}-(($ pyridin-2-yl)-methylene)-benzohydrazide) (refs. 10 and 22), slightly decrease with the spin transition from the HS to the LS state (from $109.4^{\circ}$ to $108.8^{\circ}$ and from $108.5^{\circ}$ to $107.8^{\circ}$, respectively). We could not obtain the crystal structure of $\mathrm{HS} \mathrm{Fe}(\mathrm{II})$ hydrazone complexes with protonated Hyd-N. Given the $\mathrm{NN}_{\mathrm{Hyd}} \mathrm{C}$ angle in protonated $\mathrm{HS} \mathrm{Mn}(\mathrm{II})$ and $\mathrm{Ni}(\mathrm{II})$ complexes, the $\mathrm{NN}_{\mathrm{Hyd}} \mathrm{C}$ angle centered at protonated Hyd-N in the HS Fe(II) complex would be approximately $109^{\circ}$. Furthermore, to the best of our knowledge, the literature contains no reports of protonated LS Fe(II) hydrazone complexes. From the aforementioned dependence of the $\mathrm{NN}_{\mathrm{Hyd}} \mathrm{C}$ angle on the spin state, the $\mathrm{NN}_{\mathrm{Hyd}} \mathrm{C}$ angle in the protonated LS Fe(II) hydrazone complex is expected to be only slightly smaller than that in the protonated HS Fe(II) hydrazone complex. Taking together, these considerations indicate that the $\mathrm{NN}_{\mathrm{Hyd}} \mathrm{C}$ angle at the protonated Hyd-N in the $\mathrm{Fe}(\mathrm{II})$ hydrazone complex would be approximately $114^{\circ}$ and that the $\mathrm{NN}_{\mathrm{Hyd}} \mathrm{C}$ angle at the nonprotonated Hyd-N would be approximately $109^{\circ}$. 
Table S5. Coordination distances, angles, and intramolecular hydrogen bonding parameters from the calculated results for $[\mathbf{1 C l}]_{\mathbf{H y d}}$, $[1 \mathrm{Cl}]_{\mathbf{P y}},[\mathbf{1 O M e}]_{\mathbf{H y d}}$ and $[\mathbf{1 O M e}]_{\mathbf{P y}}$ in the LS and HS states. The labels corresponding to the distances and angles in the ligand showing intramolecular proton transfer are marked with $(*)$ for clarity.

\begin{tabular}{|c|c|c|c|c|c|c|c|c|}
\hline \multirow[b]{2}{*}{$\begin{array}{c}\text { Proton Position } \\
\text { and } \\
\text { Spin State }\end{array}$} & \multicolumn{4}{|c|}{$[1 \mathrm{Cl}]$} & \multicolumn{4}{|c|}{ [1OMe] } \\
\hline & ${ }^{\mathrm{LS}}[\mathbf{1 C l}] \mathrm{Py}$ & ${ }^{\mathrm{HS}}[1 \mathrm{Cl}] \mathrm{Py}$ & $\left.\mathrm{LS}_{[1 \mathrm{Cl}}\right]_{\mathrm{Hyd}}$ & ${ }^{\mathrm{HS}}[1 \mathrm{Cl}]_{\mathrm{Hyd}}$ & ${ }^{\mathrm{LS}}[1 \mathrm{OM}] \mathrm{Py}$ & ${ }^{\mathrm{HS}}[1 \mathrm{OMe}] \mathrm{Py}$ & ${ }^{\mathrm{LS}}$ [10Me] & {$[10 \mathrm{Oe}]_{\mathrm{Hyd}}$} \\
\hline & \multicolumn{8}{|c|}{ Coordination distances around $\mathrm{Fe}(\mathrm{II})(\AA)$} \\
\hline *Fe1-N1 & 1.985 & 2.180 & 1.988 & 2.196 & 1.985 & 2.189 & 1.982 & 2.200 \\
\hline${ }^{*} \mathrm{Fe} 1-\mathrm{N} 2$ & 1.872 & 2.125 & 1.889 & 2.146 & 1.867 & 2.127 & 1.893 & 2.163 \\
\hline${ }^{*} \mathrm{Fe} 1-\mathrm{O} 1$ & 1.977 & 2.080 & 1.998 & 2.125 & 1.970 & 2.058 & 1.989 & 2.123 \\
\hline Fe1-N5 & 1.986 & 2.217 & 1.985 & 2.199 & 1.980 & 2.212 & 1.982 & 2.191 \\
\hline Fe1-N6 & 1.896 & 2.150 & 1.891 & 2.145 & 1.903 & 2.162 & 1.893 & 2.163 \\
\hline \multirow[t]{2}{*}{$\mathrm{Fe} 1-\mathrm{O} 2$} & 1.998 & 2.165 & 2.000 & 2.145 & 1.989 & 2.150 & 1.989 & 2.098 \\
\hline & \multicolumn{8}{|c|}{$\mathrm{CNPy}_{\mathrm{C}} \mathrm{C}$ angle $\left({ }^{\circ}\right)$} \\
\hline${ }^{*} \mathrm{C} 7-\mathrm{N} 4-\mathrm{C} 11$ & 124.1 & 124.1 & 119.0 & 118.8 & 123.9 & 123.7 & 118.4 & 118.3 \\
\hline \multirow[t]{2}{*}{$\mathrm{C} 25-\mathrm{N} 8-\mathrm{C} 29$} & 119.0 & 118.8 & 119.0 & 118.9 & 118.3 & 118.3 & 118.4 & 118.2 \\
\hline & \multicolumn{8}{|c|}{$\mathrm{NN}_{\mathrm{Hyd}} \mathrm{C}$ angle $\left({ }^{\circ}\right)$} \\
\hline *N2-N3-C12 & 109.9 & 110.7 & 114.2 & 115.2 & 110.1 & 110.7 & 114.1 & 115.3 \\
\hline \multirow[t]{2}{*}{ N6-N7-C30 } & 114.2 & 116.0 & 114.1 & 115.5 & 114.0 & 115.7 & 114.1 & 114.9 \\
\hline & \multicolumn{8}{|c|}{ Intramolecular hydrogen bond parameter $(\AA)$} \\
\hline$* \mathrm{~N} 3 \cdot \cdot \mathrm{N} 4$ & 2.584 & 2.566 & 2.663 & 2.678 & 2.547 & 2.532 & 2.756 & 2.734 \\
\hline$* \mathrm{~N} 3 \cdot \cdot \mathrm{H} 1$ & 1.733 & 1.700 & 1.034 & 1.031 & 1.658 & 1.631 & 1.027 & 1.028 \\
\hline$* \mathrm{~N} 4 \cdot \cdot \mathrm{H} 1$ & 1.049 & 1.055 & 1.847 & 1.902 & 1.061 & 1.070 & 2.047 & 2.026 \\
\hline $\mathrm{N} 7 \cdot \cdot \mathrm{N} 8$ & 2.660 & 2.676 & 2.665 & 2.663 & 2.742 & 2.734 & 2.756 & 2.788 \\
\hline $\mathrm{N} 7 \cdot \cdot \mathrm{H} 2$ & 1.034 & 1.031 & 1.035 & 1.033 & 1.029 & 1.028 & 1.027 & 1.026 \\
\hline $\mathrm{N} 8 \cdot \cdot \mathrm{H} 2$ & 1.870 & 1.897 & 1.887 & 1.888 & 2.028 & 2.027 & 2.047 & 2.100 \\
\hline
\end{tabular}


(1) Patel, R. N.; Sondhiya, V. P.; Patel, D. K.; Shukla, K. K.; Singh, Y. Synthesis, crystal structure, spectroscopic and superoxide dismutase activity of copper(II) and nickel(II) complex of N'-phenyl(pyridin-2-yl)methylidene benzohydrazone. Indian. J. Chem. A. 2012, 51, 1695-1700.

(2) Patel, R. N.; Singh, Y.; Singh, Y. P.; Butcher, R. J. Synthesis, crystal structure and DFT calculations of octahedral nickel(II) complexes derived from N'-[(E)-phenyl(pyridin-2-yl)methylidene]benzohydrazide. J. Coord. Chem. 2016, 69, $2377-2390$.

(3) Koo, B. K. Synthesis and Crystal Structures of Ni(II)/(III) and Zn(II) Complexes with Schiff Base Ligands. Bull. Korean Chem. Soc. 2013, 57, 859-864.

(4) Bernhardt, P. V.; Wilson, G. J.; Sharpe, P. C.; Kalinowski, D. S.; Richardson, D. R. Tuning the antiproliferative activity of biologically active iron chelators: characterization of the coordination chemistry and biological efficacy of 2-acetylpyridine and 2benzoylpyridine hydrazone ligands. J. Biol. Inorg. Chem. 2008, 13, 107-119.

(5) Despaigne, A. A. R.; Silva, J. G. D.; Carmo, A. C. M. d.; Piro, O. E.; Castellano, E. E.; Beraldo, H. Structural studies on zinc(II) complexes with 2-benzoylpyridine-derived hydrazones. Inorg. Chim. Acta. 2009, 362, 2117-2122.

(6) Singh, P.; Singh, D. P.; Tiwari, K.; Mishra, M.; Singh, A. K.; Singh, V. P. Synthesis, structural investigations and corrosion inhibition studies on $\mathrm{Mn}(\mathrm{II}), \mathrm{Co}(\mathrm{II}), \mathrm{Ni}(\mathrm{II}), \mathrm{Cu}(\mathrm{II})$ and $\mathrm{Zn}(\mathrm{II})$ complexes with 2-amino-benzoic acid (phenyl-pyridin-2-ylmethylene)-hydrazide. RSC Advances 2015, 5, 45217-45230.

(7) Bikas, R.; Hosseini-Monfared, H.; Siczek, M.; Gutiérrez, A.; Krawczyk, M. S.; Lis, T. Syntheses, crystal structures and magnetic studies of new 2D coordination polymers containing dinuclear manganese(II) repetitive units using a ditopic isonicotinhydrazone based $\mathrm{N}, \mathrm{N}, \mathrm{O}$-donor ligand. Polyhedron 2014, 67, 396-404.

(8) Khandar, A. A.; Afkhami, F. A.; Hosseini-Yazdi, S. A.; Lipkowski, J.; Dougherty, W. G.; Kassel, W. S.; Prieto, H. R.; García-Granda, S. Synthesis, Characterization and Crystal Structure of Zn(II) and Cd(II) One- and Two-Dimensional Coordination Polymers Derived from Pyridine Based Schiff Base ligand. J. Inorg. Organoment. P. 2015, $25,860-868$.

(9) Afkhami, F. A.; Khandar, A. A.; Mahmoudi, G.; Amini, M.; Molins, E.; Garczarek, P.; Lipkowski, J.; White, J. M.; Kirillov,

A. M. New cadmium(II) and zinc(II) coordination polymers derived from a pyridine-hydrazone block: Self-assembly generation, structural and topological features, and theoretical analysis. Inorg. Chim. Acta. 2017, 458, 68-76.

(10) Zhang, L.; Xu, G.-C.; Xu, H.-B.; Mereacre, V.; Wang, Z.-M.; Powell, A. K.; Gao, S. Synthesis, magnetic and photomagnetic study of new iron(II) spin-crossover complexes with $\mathrm{N}_{4} \mathrm{O}_{2}$ coordination sphere. Dalton Trans. 2010, 39, 4856-4868.

(11) Ray, A.; Banerjee, S.; Sen, S.; Butcher, R. J.; Rosair, G. M.; Garland, M. T.; Mitra, S. Two Zn(II) and one Mn(II) complexes using two different hydrazone ligands: spectroscopic studies and structural aspects. Struct. Chem. 2008, 19 (2), 209 - 217

(12) Sy, A.; Dieng, M.; Thiam, I. E.; Gaye, M.; Retailleau, P. Dichlorido $\left\{N^{\prime}-[\mathrm{phenyl}(\mathrm{pyridin}-2-\mathrm{yl}-\right.$ $\kappa N)$ methylidene]isonicotinohydrazide- $\kappa^{2} N^{\prime}, O$ \}zinc. Acta Crystallogr. E. 2013, 69, m108.

(13) Patel, R. N.; Singh, A.; Sondhiya, V. P.; Singh, Y.; Shukla, K. K.; Patel, D. K.; Pandey, R. Synthesis, characterization, and biological activity of nickel(II) complexes with a tridentate Schiff base derived from heterocyclic aldehyde. J. Coord. Chem. 2012, $65,795-812$.

(14) Banerjee, S.; Ray, A.; Sen, S.; Mitra, S.; Hughes, D. L.; Butcher, R. J.; Batten, S. R.; Turner, D. R. Pseudohalide-induced structural variations in hydrazone-based metal complexes: Syntheses, electrochemical studies and structural aspects. Inorg. Chim. Acta. 2008, 361, 2692-2700.

(15) Vantomme, G.; Jiang, S.; Lehn, J.-M. Adaptation in Constitutional Dynamic Libraries and Networks, Switching between Orthogonal Metalloselection and Photoselection Processes. J. Am. Chem. Soc. 2014, 136, 9509-9518.

(16) Patel, R. N.; Singh, A.; Shukla, K. K.; Patel, D. K.; Sondhiya, V. P. Synthesis and characterization of a new nickel(II) mixed ligand complex with 2-(2 '-pyridyl) benzothiazole. Indian. J. Chem. A. 2010, 49, 1601-1606.

(17) Das, K.; Sinha, C.; Datta, A.; Revaprasadu, N. Synthesis and Structural Characterization of a Nickel(II) Complex with Unsymmetrical NNO-Donor Schiff Base Ligand. J. Chem. Crystallogr. 2011, 41, 1032-1035. 
(18) Naskar, S.; Biswas, S.; Mishra, D.; Adhikary, B.; Falvello, L. R.; Soler, T.; Schwalbe, C. H.; Chattopadhyay, S. K. Studies on the relative stabilities of $\mathrm{Mn}(\mathrm{II})$ and $\mathrm{Mn}(\mathrm{III})$ in complexes with $\mathrm{N}_{4} \mathrm{O}_{2}$ donor environments: crystal structures of [Mn(pybzhz) $)_{2}$ ] and $\left[\mathrm{Mn}(\mathrm{Ophsal})(\mathrm{imzH})_{2}\right] \quad \mathrm{ClO}_{4} \quad\left(\right.$ pybzhz $\quad=N$-(benzoyl)- $N^{\prime}$-(picolinylidene $)$ hydrazine, Ophsal = $N, N^{\prime}-o-$ phenylenebis(salicylideneimine), imzH = imidazole). Inorg. Chim. Acta. 2004, 357, 4257-4264.

(19) Despaigne, A. A. R.; Silva, J. G. d.; Carmo, A. C. M. d.; Sives, F.; Piro, O. E.; Castellano, E. E.; Beraldo, H. Copper(II) and zinc(II) complexes with 2-formylpyridine-derived hydrazones. Polyhedron 2009, 28, 3797-3803.

(20) Chaur, M. Dichlorido $\left\{(E)\right.$-4-dimethylamino- $N^{\prime}-[($ pyridin-2-yl)methylidene- $\kappa N]$ benzohydrazide- $\kappa O\}$ zinc. Acta Crystallogr. E. 2013, 69, m27.

(21) Bernhardt, P. V.; Chin, P.; Sharpe, P. C.; Richardson, D. R. Hydrazone chelators for the treatment of iron overload disorders: iron coordination chemistry and biological activity. Dalton Trans. 2007, 30, 3232-3244.

(22) Zhang, L.; Xu, G.-C.; Xu, H.-B.; Zhang, T.; Wang, Z.-M.; Yuan, M.; Gao, S. Abrupt spin transition around room temperature and light induced properties in $\mathrm{Fe}^{\mathrm{II}}$ complexes with $\mathrm{N}_{4} \mathrm{O}_{2}$ coordination sphere. Chem. Commun. 2010, 46, $2554-2556$.

(23) Li, L.; Zhang, Y. Z.; Liu, E.; Yang, C.; Golen, J. A.; Rheingold, A. L.; Zhang, G. Synthesis and structural characterization of zinc(II) and cobalt(II) complexes based on multidentate hydrazone ligands. J. Mol. Struct. 2016, 1110, $180-184$.

(24) Mahmoudi, G.; Chowdhury, H.; Lofland, S. E.; Ghosh, B. K.; Kirillov, A. M. Two manganese(II) coordination polymers driven by (iso)nicotinoyl-hydrazone blocks and pseudohalide ancillary ligands: syntheses, structural features, and magnetic properties. J. Coord. Chem. 2017, 70, 1973-1983.

(25) Jang, Y. J.; Lee, U.; Koo, B. K. Synthesis and crystal structures of Mn(II), Co(II), Ni(II), Cu(II), and Zn(II) metal complexes with NNO functionalized ligands. Bull. Korean Chem. Soc. 2005, 26, 925-929.

(26) Das, K.; Sinha, C.; Datta, A. Structural Aspects and Spectroscopic Characterization of a Mn(II) Complex with Unsymmetrical NNO-Donor Hydrazone Ligand. J. Chem. Crystallogr. 2012, 42, 150-154.

(27) Datta, A.; Chuang, N.-T.; Sie, M.-H.; Huang, J.-H.; Lee, H. M. Bis $\left\{N^{\prime}-[1\right.$-(2-pyridyl)ethylidene- $\kappa N]$ benzohydrazidato$\kappa^{2} N^{\prime}, O$ \}nickel(II). Acta Crystallogr. E. 2010, 66, m359.

(28) Mangalam, N. A.; Sheeja, S. R.; Kurup, M. R. P. Mn(II) complexes of some acylhydrazones with NNO donor sites: Syntheses, a spectroscopic view on their coordination possibilities and crystal structures. Polyhedron 2010, 29, $3318-3323$.

(29) Bakir, M.; Green, O.; Mulder, W. H. Synthesis, characterization and molecular sensing behavior of $\left[\mathrm{ZnCl}_{2}\left(\eta^{3}-\mathrm{N}, \mathrm{N}, \mathrm{O}-\right.\right.$ dpkbh) $($ dpkbh = di-2-pyridyl ketone benzoyl hydrazone). J. Mol. Struct. 2008, 873, 17-28.

(30) Bakir, M.; Conry, R. Synthesis, spectroscopic and X-ray crystallographic properties of manganese compounds of keto- and enol-coordinated di-2-pyridyl ketone benzoyl hydrazone (dpkbh). Reactions of $\mathrm{Mn}(\mathrm{CO})_{5} \mathrm{Br}$ with dpkbh. J. Coord. Chem. 2016, 69, 1244-1257.

(31) Bernhardt, P. V.; Mattsson, J.; Richardson, D. R. Complexes of cytotoxic chelators from the dipyridyl ketone isonicotinoyl hydrazone (HPKIH) analogues. Inorg. Chem. 2006, 45, 752-760.

(32) Bernhardt, P. V.; Caldwell, L. M.; Chaston, T. B.; Chin, P.; Richardson, D. R. Cytotoxic iron chelators: characterization of the structure, solution chemistry and redox activity of ligands and iron complexes of the di-2-pyridyl ketone isonicotinoyl hydrazone (HPKIH) analogues. J. Biol. Inorg. Chem. 2003, 8, 866-880. 